\section{Multifocal Skeletal Tuberculosis in 2 Immunocompetent Children}

\section{To the Editor:}

Tuberculosis remains a worldwide major global health problem. Osteoarticular tuberculosis represents $1 \%-2 \%$ of cases. Multifocal skeletal forms are exceptional even in endemic countries and affect mainly immunocompromised patients. It is usually associated with a primary pulmonary tuberculosis ${ }^{1,2}$. Clinical presentation is often misleading and contributes to a delay in diagnosis. We describe 2 cases of multifocal skeletal tuberculosis in young immunocompetent patients, both coming from Africa and living in France for 3 years.

Case 1. A 12-year-old girl, a native of Ghana, presented with a 3-month history of lumbar pain and rapid-onset S1 left radicular distribution. She had a history of asthenia with weight loss of $3 \mathrm{~kg}$ without fever. On examination, she had tenderness over the thoracolumbar spine, with muscle spasm and stiffness. Lasegue's sign was positive without any neurologic deficiency. Imaging [plain radiographs, computed tomography (CT), magnetic resonance imaging (MRI)] revealed lytic lesions of the ninth and tenth ribs, wedging of the body of T12 with soft-tissue edema, and bone destruction of T11, S1, and right sacral ala associated with a 6-cm collection in the pelvic cavity (Figures 1 and 2). Radiographs of the chest were unremarkable.

There was a raised erythrocyte sedimentation rate (ESR) of $51 \mathrm{~mm} / \mathrm{h}$. The tuberculin test was phlyctenular. Histopathological examination of a sacrum bone biopsy revealed granulomatous lesions with caseous material and multinucleated giant epithelioid cells. Staining and polymerase chain reaction (PCR) were both negative but tissue culture disclosed a Mycobacterium africanum tuberculosis infection leading to the diagnosis of a multifocal skeletal form. Antituberculous chemotherapy and spinal bracing were initiated. Despite this treatment, compression of L4, L5, and S1 from an expanding abscess appeared 2 weeks later. Emergency surgery was done, consisted of drainage of the abscess and anterior spine fusion with bone grafting. The patient recovered without sequelae.

Case 2. A 16-year-old girl, born in Senegal, presented with a sudden right-leg paralysis. She appeared to be in good health and afebrile. She had been complaining of inflammatory dorsalgia for 3 months. On examination, she had stiffness on the thoracolumbar spine with neurological motor and sensory deficit of the right leg. Radiographs, CT scan, and MRI of the spine showed a wedging of T11 with spinal cord compression and lytic lesions of T4, T8, T9, T11, T12, L2, and L4. Chest radiograph was normal. ESR was elevated $(82 \mathrm{~mm} / \mathrm{h})$. No tuberculosis skin test was done because of the unknown vaccine status of the patient. A T10-T12 laminectomy was performed. Microbiological examinations were negative. Bone biopsy of L4 revealed granulomatous inflammation with caseous necrosis and giant epithelioid cells. Ziehl's staining and PCR were negative but multifocal tuberculosis due to M. tuberculosis was confirmed by culture. Pharmaceutical antitubercular treatment was administered, with spinal bracing and drainage of the T11 paravertebral abscess. Secondary posterior spinal fusion was chosen because of progressive T11 instability.

Although tuberculosis has a low incidence in European countries, North America, and Australia, it remains frequent among newly arrived immigrants from endemic countries $(<2$ years $)$, immunocompromised patients, the homeless, and the elderly ( $>80$ years old).

Osteoarticular tuberculosis is rare and is usually seen in endemic countries $^{3}$. Children between 1 and 10 years old are often affected, constituting $50 \%$ of cases, with a higher incidence in boys ${ }^{4,5}$. The multifocal form is exceptional; 1 location is found in $90 \%-95 \%$ of cases. It is most often seen in disseminated disease with concomitant pulmonary infection (45\% in the first decade, $10 \%$ to $20 \%$ in patients older than 10 years $)^{6}$. Of patients with skeletal tuberculosis, $50 \%$ have spine lesions, $30 \%$ hip or knee lesions, and $10 \%$ sacroiliac lesions ${ }^{3,7}$. Involvement of ribs and sacral ala is unusual and accounts for $<1 \%$ of all cases of osteoarticular tuberculosis ${ }^{5}$. The most

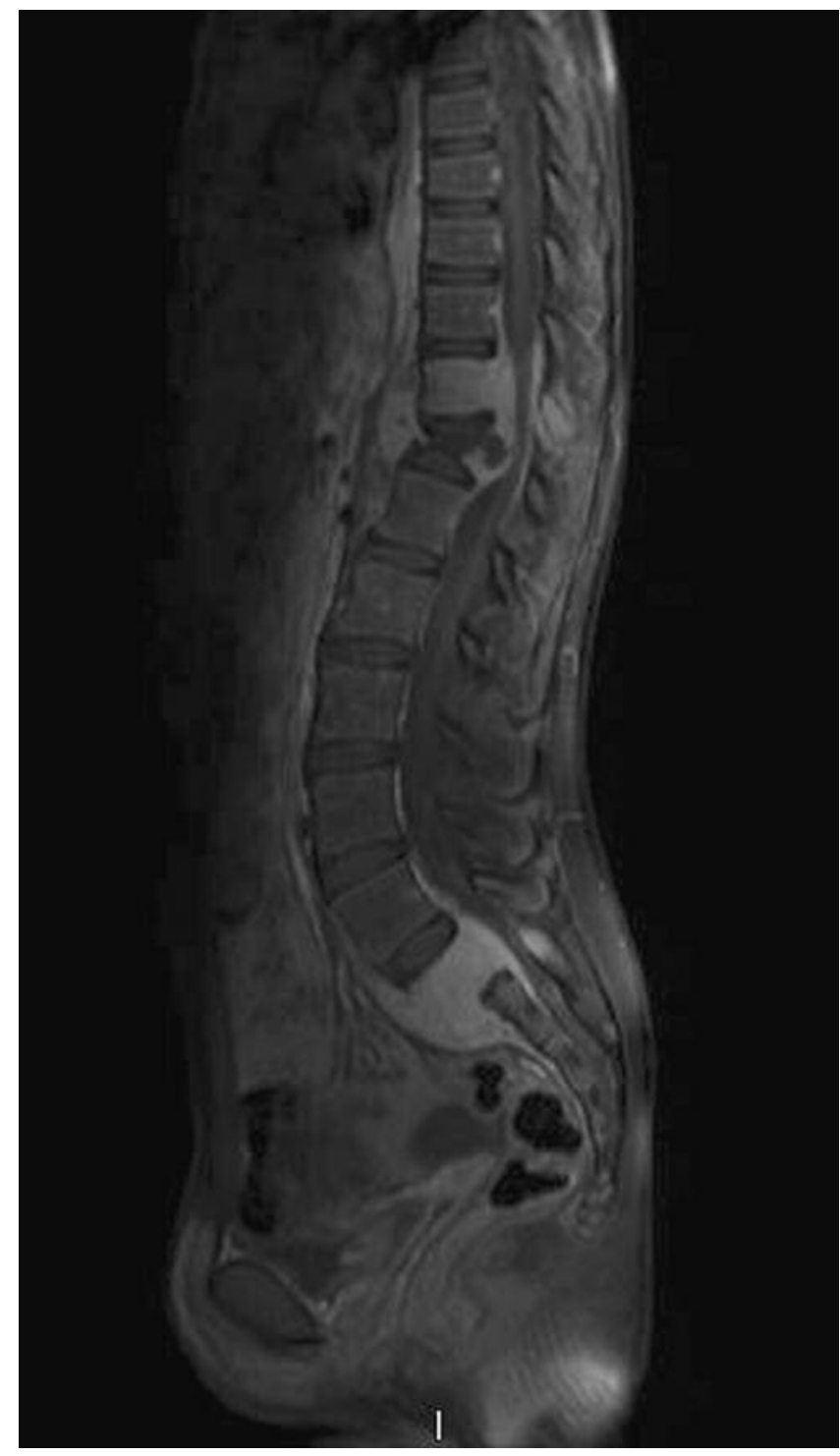

Figure 1. Sagittal T1-weighted MRI of the spine shows marked destruction of T11, T12, and S1 vertebral bodies with large compressive paravertebral abscesses.

commonly reported complications are expanding abscesses, spinal cord compression, kyphotic deformity, and spine instability.

Knowledge of personal history is essential to reach this diagnosis. A tuberculin test may be falsely negative. Imaging is useful to detect early involvement and to define the extent of the disease. While histologic examination shows a granulomatous lesion suggestive of tuberculosis, evidence of infection by direct examination and tissue culture study or PCR confirms the diagnosis.

Although M. tuberculosis is responsible for the major part of tuberculosis infection, our first case was related to M. africanum. This species is most commonly found in West African countries and has a lower pathogenicity, developing mainly in immunocompromised patients.

Antituberculous therapy has to be initiated quickly in order to avoid sequelae. Surgery is needed only if there is a neurologic deficit or spinal instability. Abscesses require surgical drain.

These 2 exceptional cases show that tuberculosis must be considered as a differential diagnosis in cases with multiple destructive bone lesions in 


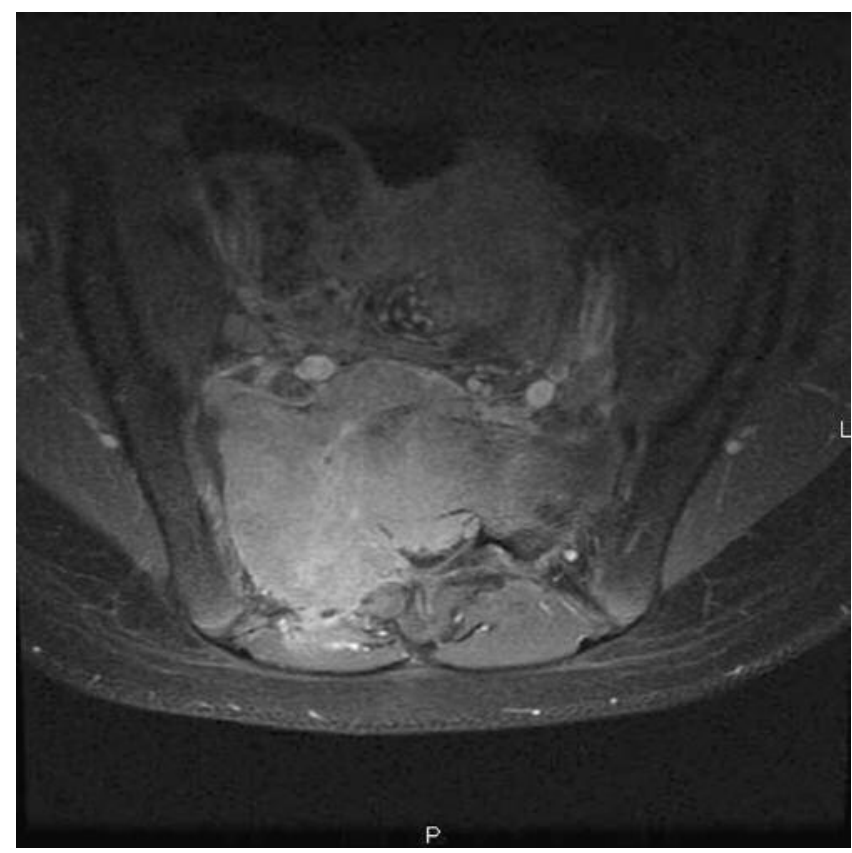

Figure 2. Axial postcontrast T1-weighted MRI showing S1 and right lateral part of the sacrum destruction with extensive abscess in the pelvic cavity.

immunocompetent or immunocompromised patients coming from endemic countries.

CAROLINE CAPUANI, MS; FRANCK ACCADBLED, MD, PhD,

Orthopedic Surgery; MARIE-BERNADETTE DELISLE, MD, PhD, Pathology, CHU Rangueil, 1 Avenue Jean Poulhès, TSA 50032 31059, Toulouse 31059, France; ANNE GOMEZ-BROUCHET, MD, PhD; Hôpital des enfants, CHU-Purpan, Toulouse. Address correspondence to Dr. Gomez-Brouchet; E-mail: Brouchet.anne@chu-toulouse.fr

\section{REFERENCES}

1. Hong L, Wu JG, Ding JG, Wang XY, Zheng MH, Fu RQ, et al. Multifocal skeletal tuberculosis: Experience in diagnosis and treatment. Med Mal Infect 2010;40:6-11.

2. Marudanayagam A, Gnanadoss JJ. Multifocal skeletal tuberculosis: a report of three cases. Iowa Orthop J 2006;26:151-3.

3. Gelal F, Sabah D, Dogan R, Avci A. Multifocal skeletal tuberculosis involving the lumbar spine and a sacroiliac joint: MR imaging findings. Diagn Interv Radiol 2006;12:139-41.

4. Tiwari A, Sud A, Mehta S, Kanojia RK, Kapoor SK. Multifocal skeletal tuberculosis presenting as multiple bone cysts. Ann Acad Med Singapore 2007;36:1038-9.

5. Morris BS, Varma R, Garg A, Awasthi M, Maheshwari M. Multifocal musculoskeletal tuberculosis in children: appearances on computed tomography. Skeletal Radiol 2002;31:1-8.

6. Goldblatt M, Cremin BJ. Osteo-articular tuberculosis: its presentation in coloured races. Clin Radiol 1978;29:669-77.

7. Le Roux P, Quinque K, Bonnel AS, Le Luyer B. Extra-pulmonary tuberculosis in childhood. Arch Pediatr 2005;12 Suppl 2:S122-6.

J Rheumatol 2010;37:11; doi:10.3899/jrheum.100562 